OPEN ACCESS

Edited by:

Fabienne Brilot,

University of Sydney, Australia

Reviewed by:

David Brown,

Westmead Institute for Medical

Research, Australia

Enrico Castroflorio,

Medical Research Council Harwell

(MRC), United Kingdom

*Correspondence:

Mette Scheller Nissen

mette.scheller.nissen2@rsyd.dk

Specialty section:

This article was submitted to

Multiple Sclerosis and

Neuroimmunology,

a section of the journal

Frontiers in Neurology

Received: 06 July 2019 Accepted: 18 September 2019

Published: 02 October 2019

Citation:

Nissen MS and Blaabjerg M (2019) Anti-IgLON5 Disease: A Case With 11-Year Clinical Course and Review of the Literature. Front. Neurol. 10:1056. doi: 10.3389/fneur.2019.01056

\section{Anti-IgLON5 Disease: A Case With 11-Year Clinical Course and Review of the Literature}

\author{
Mette Scheller Nissen ${ }^{1,2,3 *}$ and Morten Blaabjerg ${ }^{1,2,3}$ \\ ${ }^{1}$ Department of Neurology, Odense University Hospital, Odense, Denmark, ${ }^{2}$ Department of Clinical Research, University of \\ Southern Denmark, Odense, Denmark, ${ }^{3}$ BRIDGE, Brain Research - Inter-Disciplinary Guided Excellence, University of \\ Southern Denmark, Odense, Denmark
}

Background: Anti-IgLON5 disease is a novel disorder with a complex interplay between inflammation and neurodegeneration. Patients develop antibodies against IgLON5 but also deposition of neuronal tau protein. Symptoms often have an insidious onset, slow progression and mimic other neurological disorders. Here we report a case with severely prolonged 11-year disease course and provide a review of current reported cases with focus on presentation, work-up, treatment, and outcome.

Method: All reported cases of anti-IgLON5 disease were evaluated. Cases reported twice (in case series and as single case reports), were carefully excluded.

Results: Most patients display a characteristic sleep disorder with severe insomnia, non rapid eye movement (NREM) parasomnia, with finalistic movements and sleep disordered breathing (stridor and obstructive sleep apnea). Other symptoms are bulbar involvement, gait instability, movement disorders, oculomotor abnormalities, dysautonomia, and peripheral symptoms. Antibodies are present in both serum and CSF and there is a strong correlation with human leukocyte antigen (HLA) DRB1*10:01 and HLA-DQB1*05:01. Neuropathological examination reveals neurodegeneration with neuronal tau deposits in regions that correlate with the clinical presentation (e.g., predominantly hypothalamus and tegmentum of the brain stem). Majority of cases respond partially to immunotherapy. Cases, who received no treatment or treatment with IV corticosteroids alone, had a higher mortality than cases treated with more potent immunotherapy.

Conclusion: The clinical spectrum of Anti-IgLON5 disease continues to expand. Further studies are needed to elucidate the pathophysiology, therapeutic strategies and outcome in this novel disorder. Aggressive immunotherapy seems to increase survival.

Keywords: autoimmune encephalitis, IgLON5, inflammation, tau, immunology

\section{BACKGROUND}

The recently described disease with antibodies against immunoglobulin-like cell adhesion molecule 5 (IgLON5), is characterized by a distinctive sleep disorder associated with a broad variety of neurological symptoms such as gait instability, movement disorders, and brainstem involvement (1). Antibodies against IgLON5 have been described to cause irreversible internalization of surface IgLON5 and postmortem studies have demonstrated deposits of hyperphosphorylated 
tau (p-tau), with predominant involvement of hypothalamus and tegmentum of the brainstem, but also hippocampal formation and cerebellum $(2,3)$. Strong association with human leukocyte antigen (HLA) DRB1*10:01 and HLA-DQB1*05:01 alleles has been reported, making the IgLON5 disease a complex interaction between neurodegeneration and neuroimmunology with a genetic predisposition (4). Anti-IgLON5 disease differs from previous described autoimmune encephalitis (AIE) syndromes by a protracted clinical disease course, deposition of tau and a variable effect of immunotherapy, making it a challenge to diagnose and treat $(4,5)$. Since 2014, more than 60 cases have been reported, expanding the spectrum of neurological symptoms (4-19). Here we present a case report displaying a severely prolonged 11-year disease course and review of the current literature, focusing on clinical presentation, work-up, treatment and outcome of patients with anti-IgLON5 disease.

\section{METHODS}

All cases and case series were thoroughly examined. One case series, focusing on post-mortem findings, reported three cases previously described, and three cases with "probable" antiIgLON5 disease due to neuropathological findings, but with unknown antibody status (3). These three probable cases, were not included in this review. One case series included previously published single cases and small case series (4). These duplicated cases were carefully excluded. We thus ended up with a review of 58 cases including our own.

The presented case provided informed consent for publication.

\section{CASE}

In January 2019, a 61-year old male, with a 1-year history of diagnosed obstructive sleep apnea (OSA), was admitted in a state of unconsciousness due to hypercapnia. He had an 11-year history of slowly progressive diplopia, hoarseness, slurred speech, dysphagia and sleep disturbances. At disease onset the initial symptoms were diplopia and mild dysphagia. On suspicion of multiple sclerosis a brain MRI was performed. It showed T2 weighted unspecific white matter (WM) hyperintensities in the brainstem. Cerebrospinal fluid (CSF) analysis revealed mild pleocytosis (15 white blood cells/uL), but normal protein levels and no oligoclonal bands (OCB). Visual and somatosensory evoked potential (VEP/SSEP) were normal. Multiple subsequent brain MRI's showed no further progression of the WM hyperintensities.

In 2018 he developed respiratory symptoms and was diagnosed with OSA (Apnea Hypopnea Index: 25). Despite continuous positive airway pressure (CPAP) treatment, he was admitted several times with respiratory failure at night within the last year, and his wife reported sleep abnormalities with atypical movements. Within the last 6 months symptoms progressed and he developed mild gait imbalance and behavioral changes with disinhibition.
Neurological examination revealed horizontal gaze palsy, ptosis of the left eyelid, mild dysarthria, oro-facio-mandibular dystonia, and mild tetraparesis with spasticity and Babinski's sign in his lower right extremity. Moreover, a mild gait ataxia and fasciculations on both upper and lower extremities were noticed.

Laryngoscopy was performed showing bilateral vocal cord palsy. Whole-body 18-FDG PET CT scan, electromyography (EMG), and nerve conduction (ENG) studies showed no abnormalities. Acetylcholine receptor antibodies were negative. A Mini Mental State Examination showed mild cognitive impairment (24/30) with visuospatial abnormalities.

CSF analysis was normal. Because of the slowly progressive symptoms including sleep disturbances, OSA, bulbar symptoms and gait imbalance, antibodies against IgLON5 were tested and found strongly positive in both serum and CSF (1:1,000 and 1:10 CBA Euroimmun) confirming the diagnosis. Analysis for $\mathrm{IgG}$ isotypes showed predominantly IgG4 isotype antibodies and the patient was HLA DRB1*10:01 and HLA-DQB1*05:01 positive (kindly performed by Dr. Gaig and colleagues).

Immunotherapy was initiated with high dose intravenous corticosteroids and therapeutic plasma exchange (TPE). Subsequent polysomnography (PSG) showed severe insomnia with overall $24 \mathrm{~min}$ of sleep, a sleep efficiency of $4 \%$, stridor, vocalizations and abnormal movements both during wakefulness and sleep. Neither N3 sleep stages nor REM sleep was present. The patient and his spouse reported subjective improvement on gait imbalance and sleep, but no significant clinical improvement was found on neurologic examination. Second line treatment with Rituximab was initiated.

Three weeks later, he developed hypercapnia and respiratory failure during daytime, requiring mechanical ventilation. Repeated antibody analysis still showed strongly positive antiIgLON5 antibodies in serum (1:100) but only mildly positive in CSF (1:1). Renewed TPE was initiated. At time of review the patient was fully awake, mRS score 2 , but had undergone tracheotomy and received assisted mechanical ventilation at night. Sleep quality was improved and movements during sleep diminished.

\section{DEMOGRAPHICS AND CLINICAL PRESENTATION}

Anti-IgLON5 disease affects men and women equally and only $10 \%$ of patients have a history of autoimmune disease. In currently documented cases, median age at diagnosis was 62 years, ranging from 45 to 79 years. Only $11 \%$ had a history of malignancy, with no clear tendency of cancer type (Table 1).

Symptoms are often heterogeneous, with insidious onset and slow progression. The median time from symptom onset to diagnosis was 12 months (range 3 weeks to 11 yearsSupplementary Table 1).

The most prominent feature of the anti-IgLON5 disease is a distinctive sleep disorder, including both REM and nonREM parasomnia, finalistic movements and sleep disordered breathing with stridor and OSA (4, 5, 20). The NREM parasomnia seen in IgLON5 positive patients, differs from 
TABLE 1 | Demographics, antibody status, and CSF findings.

\begin{tabular}{lc}
\hline Demographics & No. (\%) \\
\hline Sex, female $(n=64)$ & $32(50)$ \\
Age at diagnosis $(n=35)$ (years, range) & $62(45-79)$ \\
Hx autoimmune disease $(n=58)$ & $6(10.3)$ \\
Hx of malignancy $(n=36)$ & $4(11.1)$ \\
Antibody status CSF and serum & Positive \\
CSF IgLON5 $(n=40)$ & $38(94.9)$ \\
Serum IgLON5 $(n=63)$ & $63(100)$ \\
IgG isotype, serum $(n=48)$ & \\
- IgG1 & $45(93.8)$ \\
- IgG2 & $30(62.5)$ \\
- IgG3 & $23(47.9)$ \\
- IgG4 & $44(91.7)$ \\
HLA-DRB1*10:01; DQB1*05:01 alleles $(n=26)$ & $24(92.3)$ \\
CSF findings $(\boldsymbol{n}=41)$ & No. (\%) \\
Pleocytosis $(>5$ leukocytes/ $/ \mathrm{LL})$ & $10(24.4)$ \\
Increased protein $(>45 \mathrm{mg} / \mathrm{dL})$ & $20(48.8)$ \\
Oligoclonal bands $(n=29)$ & $3(10.3)$ \\
Tau $(n=6)$ & $1(16.7)^{\star}$ \\
P-tau $(n=7)$ & $2(28.6)^{\star}$ \\
$\beta$-amyloid $(n=5)$ & $0 *$ \\
\hline
\end{tabular}

"From single case series included in Gaig et al. (4).

conventional NREM parasomnias (e.g., confusional arousals, sleep walking, sleep terrors), as it consists of poorly structured N2 sleep and undifferentiated NREM sleep (20). In cases, where detailed information was available, sleep disorder was present in $83 \%$ of patients and as part of initial symptoms in $40 \%$ (Table 2). Many patients report excessive daytime sleepiness and bed partners often complain of vocalizations, snoring and abnormal behavior and movement during sleep (20).

Apart from sleep disturbances, the most frequent symptoms at disease presentation are gait instability (e.g., imbalance and ataxia), bulbar symptoms (dysphagia, dysarthria, laryngospasm, and respiratory symptoms) and movement disorders (chorea, parkinsonism, dystonia), accounting for $>60 \%$ of symptoms (Table 2). Gait instability is often mild to moderate, and not always due to ataxia. Dysphagia is the most reported bulbar symptom (present in 67\% of cases) and patients often report mild-moderate or intermittent problems swallowing. However, some patients experience severe dysphagia, with significant weight loss requiring a percutaneous endoscopic gastrotomy. Dysarthria (present in 38\% of cases) is often characterized by hoarseness and dysphonia and accompanied by laryngospasms or vocal cord palsy. Additional often reported symptoms include oculomotor abnormalities in 43\% (vertical and horizontal gaze palsy, nystagmus, ptosis, hypometric saccades), neuropsychiatric symptoms in 50\% (cognitive impairment, memory deficits, and hallucinations), dysautonomia in $41 \%$ (urinary incontinence or urgency, constipation, anhidrosis), and symptoms of the peripheral nervous system in $32 \%$ (fasciculations, cramps, neuropathy)
TABLE 2 | Symptoms at onset vs. during disease and imaging findings.

\begin{tabular}{|c|c|c|}
\hline Symptom & $\begin{array}{l}\text { Initial symptom } \\
(n=38) \text { No. (\%) }\end{array}$ & $\begin{array}{l}\text { Present overall } \\
(n=58) \text { No. }(\%)\end{array}$ \\
\hline Sleep & 15 (39.5) & $48(82.8)^{\star}$ \\
\hline Obstructive sleep apnea & $1(2.6)$ & 34 (58.6) \\
\hline Bullbar dysfunction & $8(21.1)$ & $44(75.9)$ \\
\hline Oculomotor dysfunction & $1(2.6)$ & $25(43.1)$ \\
\hline Movement disorder & $7(18.4)$ & $26(44.8)$ \\
\hline Gait disorder & $10(26.3)$ & $37(63.8)$ \\
\hline Neuropsychiatric symptoms & $3(7.9)$ & $29(50)$ \\
\hline Peripheral nervous system & $1(2.6)$ & $12(31.6)^{\star \star}$ \\
\hline Autonomic dysfunction & 0 & $24(41.4)$ \\
\hline MRI $(n=57)$ & \multicolumn{2}{|c|}{ No. (\%) } \\
\hline Normal & \multicolumn{2}{|c|}{$46(80.7)$} \\
\hline Abnormal & \multicolumn{2}{|c|}{$11(19.3)$} \\
\hline - Brainstem atrophy & \multicolumn{2}{|c|}{3} \\
\hline - Bilat hippocampal atrophy & \multicolumn{2}{|c|}{1} \\
\hline - Cerebellar atrophy & \multicolumn{2}{|c|}{2} \\
\hline - Hypothalamic hyperintensities, T2 & \multicolumn{2}{|c|}{1} \\
\hline $\begin{array}{l}\text { - Focal enhancement of leptomeninges } \\
\text { + edema, fronto-temporal enhancement }\end{array}$ & \multicolumn{2}{|c|}{1} \\
\hline - Brainstem hyperintensities, T2 & \multicolumn{2}{|c|}{1} \\
\hline - Thalamic hyperintensity, T2 & \multicolumn{2}{|c|}{1} \\
\hline FDG PET CT $(n=8)$ & \multicolumn{2}{|c|}{ No. (\%) } \\
\hline - Normal & \multicolumn{2}{|c|}{$4(50)$} \\
\hline - Abnormal & \multicolumn{2}{|c|}{$4(50)$} \\
\hline - Striatal + brainstem hypermetabolism & \multicolumn{2}{|c|}{1} \\
\hline $\begin{array}{l}\text { - hypermetabolism basal ganglia + } \\
\text { cerebellum }\end{array}$ & \multicolumn{2}{|c|}{1} \\
\hline $\begin{array}{l}\text { - Hypermetabolism left frontal, temporal } \\
\text { lobe. bilat caudate nucleus and putamen }\end{array}$ & \multicolumn{2}{|c|}{1} \\
\hline $\begin{array}{l}\text { - Hypermetabolism sensori-motor } \\
\text { cortex, basalganglia, cerebellum }\end{array}$ & \multicolumn{2}{|c|}{1} \\
\hline DAT-SPECT $(n=1)$ & \multicolumn{2}{|c|}{ No. } \\
\hline Normal & \multicolumn{2}{|c|}{1} \\
\hline Tau-PET $(n=1)$ & \multicolumn{2}{|c|}{ No. } \\
\hline Increased tau brain stem and cerebellum & \multicolumn{2}{|c|}{1} \\
\hline TSPO-PET $(n=1)$ & \multicolumn{2}{|c|}{ No. } \\
\hline Microglia activation in leptomeninges & \multicolumn{2}{|c|}{1} \\
\hline
\end{tabular}

"Five patients had unknown sleep pattern (not available), five patients without sleep disturbances.

${ }^{*}$ Calculated from $n=37$.

(Table 2). An overview of symptoms and their association is provided in Figure 1.

The heterogenous clinical presentation of neurological symptoms makes it difficult to distinguish anti-IgLON5 disease from other neurological diseases. In a previous report, four clinical phenotypes were proposed, (I) predominant sleep disorder, (II) bulbar syndrome, (III) gait instability and oculomotor involvement resembling Progressive Supranuclear Palsy (PSP-like syndrome), and (IV) cognitive impairment with/without chorea (4). Since then, case series and cases have described various phenotypes broadening the spectrum of anti-IgLON5 disease even further. Sudden onset with acute 


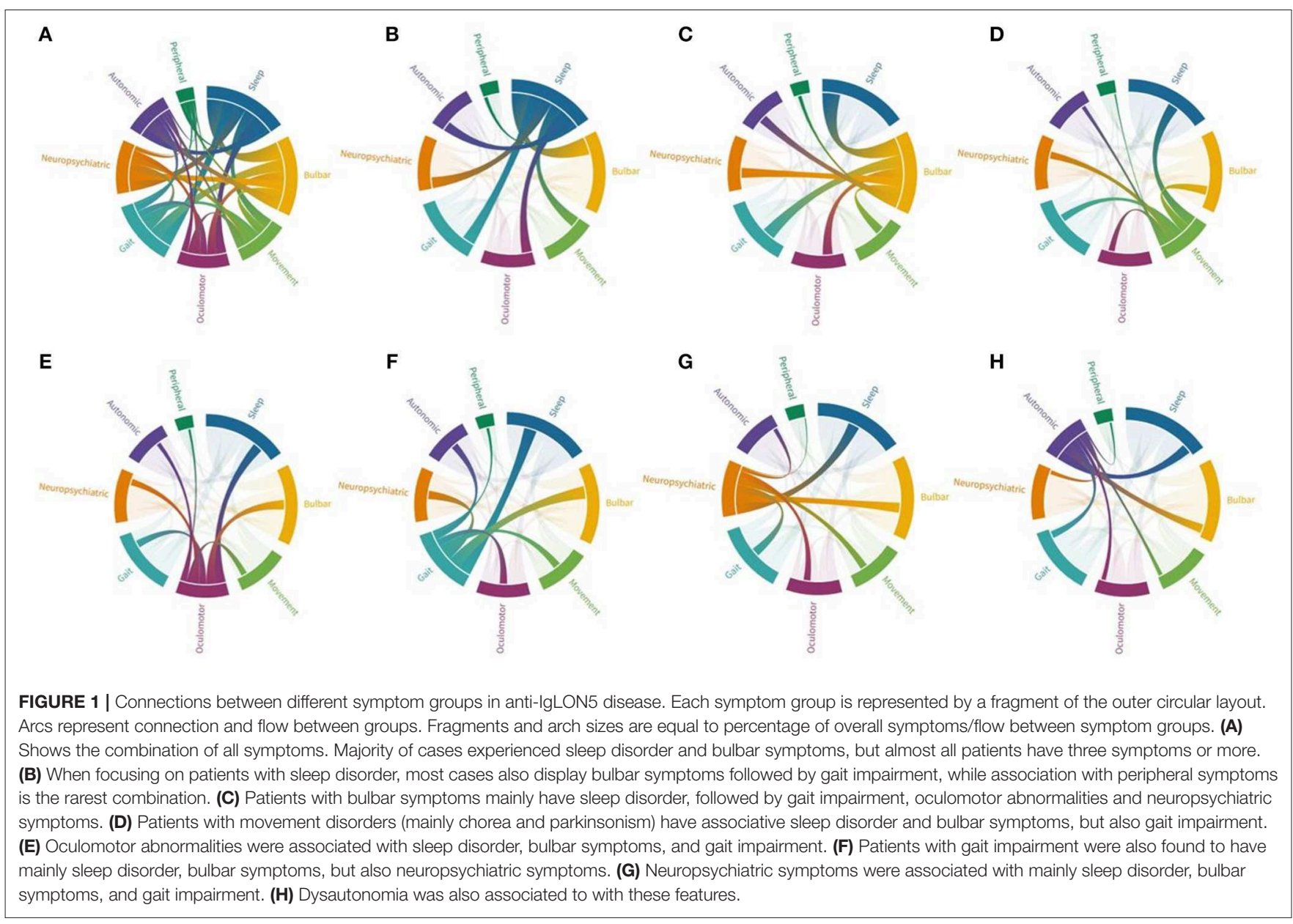

encephalic presentation and dyskinesia has been described in at least two cases $(17,18)$. One patient presented with severe muscle stiffness and hyperekplexia as seen in stiffperson syndrome (SPS) (5) and other case reports find fasciculations and peripheral symptoms such as peripheral nerve palsy, atrophy and muscle cramps (5, 9, 14, 16) (including our case). Depending on the presentation and combination of symptoms the clinical profile makes antiIgLON5 disease easily misdiagnosed. The sleep disorder could be interpreted as pure OSA or REM sleep behavior disorder. A combination of sleep disorder, bulbar symptoms, gait imbalance, and movement disorders can resemble symptoms seen in neurodegenerative diseases such as PSP or multiple system atrophy (MSA). And a clinical profile dominated by respiratory failure, autonomic dysfunction, and hyperekplexia can be interpreted as neuromuscular or motor neuron diseases (MND).

Anti-IgLON5 disease is a rare disease, with an estimated incidence of 1/150,000 (5). Several studies have investigated subpopulations (e.g., sleep, neurodegenerative, and psychiatric cohorts) without finding a significant level of anti-IgLON5 positive cases $(1,21,22)$. Thus, antibody testing in the absence of combination of abovementioned neurological symptoms is not recommendable.
The symptoms in anti-IgLON5 disease are likely explained by neuropathologic findings in post mortem studies $(1,3)$. The p-tau deposition in hypothalamic and hippocampal areas explains the characteristic sleep disorder and amnestic syndrome, and involvement of the nuclei in tegmentum of the brainstem explains gait instability, movement disorders, and oculomotor deficits.

\section{IMMUNOLOGY AND PATHOPHYSIOLOGY}

The IgLON proteins are a family of cell adhesions molecules, containing five different proteins (Opiod-binding cell adhesion molecule (IgLON1), neurotrimin (IgLON2), limbic systemassociated membrane protein (IgLON3), neuronal growth regulator 1 (IgLON4) and IgLON5). Structurally IgLONs are $\sim 340$ amino acids long and tied to the cell membrane. They possess three Ig-like domains. Through the first Ig-domain, it has recently been indicated that IgLON's can homo- and heterodimerize with high affinity and in a $\mathrm{Ca}^{2+}$ independent manner, thus making them able to interact across different cells and/or the synaptic cleft (23).

The complete function of the proteins and their interactions remain largely unclear. It is, however, known that the IgLON proteins play a role in neuronal development and synaptic 
formation (e.g., neurite sprouting and cell adhesion, astrocyte growth and limbic axonal differentiation), as well as cortical and hippocampal proliferation and synaptogenesis (2, 2426). It has been suggested that IgLONs play a role in the evolution of brain anatomy and complex maturation, influencing cellular migration and brain-blood barrier integrity (27).

Polymorphisms in the IgLON1 and IgLON2 gene have been found to be associated with late onset Alzheimers disease (28), whereas IgLON3 has been linked to schizophrenia and depression (29). This is intriguing knowledge considering the phenotype of anti-IgLON5 disease with prominent symptoms such as dementia and behavioral symptoms, alongside tau depositions, and may further strengthen the hypothesis that damage to the IgLON5 protein is an underlying mechanism of the disease.

Patients with anti-IgLON5 disease display all four isotypes of immunoglobulin G (IgG) but predominantly IgG1 (94\%) and IgG4 (92\%), with a higher density of IgG4 than IgG1 in serum (Table 1).

The role of anti-IgLON5 antibodies in autoimmunity is an ongoing discussion. The main antibody target on IgLON5 is the Ig-like domain 2 and the IgG1 anti-IgLON5 antibody population has been shown to cause irreversible pathological internalization of IgLON5, supporting the immune mediated theory of the disease (2). However, majority of antibodies are IgG4, which have not been found to induce endocytosis of IgLON5 (2). It cannot be ruled out, that IgG4 antibodies may exert an effect on IgLON5 protein function or stability (as seen in other IgG4 antibody mediated AIE, such as LGI1 or Caspr2), this however remains to be further elucidated $(2,30)$.

Anti-IgLON5 disease differs from other AIEs because the assumed immunologic pathology is described in combination with neurodegeneration shown in neuropathological investigations. The main neuropathological findings are abnormal p-tau protein deposits (3-repeat and 4-repeat isoforms) in neurons, with no concurrent inflammatory infiltrates, beta-amyloid or alpha-synuclein deposits. Tau aggregates were predominantly found in the hypothalamus and tegmentum of the brainstem with a rostrocaudal gradient involving hippocampus, medulla oblongata, periaqueductal gray matter and the upper cervical cord $(1,3)$. Until now, only sparse glial and white matter involvement has been described. Microglia activation has been found in two cases $(12,31)$.

It is still unknown if antibodies are an epiphenomenon appearing secondary to the neurodegeneration or if the neurodegeneration is purely antibody mediated (32). But a combination of autoantibodies, a solid association to HLA haplotypes, and response to immunotherapy in some cases, supports the latter. A combination of HLA-DRB1*10:01 and HLA-DQB1*05:01, or HLA-DQB1*05:01 alone (in two cases) was found in $92 \%$ of cases of anti-IgLON5 disease, where HLA-type was tested (Table 1). The HLA-DRB1*10:01 allele is found in $<2 \%$ of the general population and increased up to 36 times in patients with anti-IgLON5 disease (4).

\section{DIAGNOSIS}

Detection of anti-IgLON5 antibodies is crucial for diagnosis. Antibodies can be detected in both serum and CSF and are positive in 95 and $100 \%$ of cases, respectively (Table $\mathbf{1}$ ).

Routine CSF findings are normal in approximately half of cases. Elevated protein level is the most frequent finding, seen in $49 \%$ of cases. Pleocytosis is found in $24 \%$ of cases, and is mild with majority of cell count levels between 5 and 10 leukocytes/ $\mu \mathrm{L}$. OCBs are rarely present. Due to the neuropathology of the disease, tau, p-tau and beta-amyloid have been measured in CSF in some recent cases $(10,13,18,19,31,33)$. However, levels of $\mathrm{p}$-tau were only increased in two out of seven cases (Table 1).

Evaluation of imaging findings revealed abnormal MRI findings in only $19 \%$ of patients. The most common finding was brainstem/cerebellar atrophy $(45 \%)$. Of note, most of changes found on MRI are in regions shown affected by the tauopathy (hypothalamus, brainstem, hippocampus, cerebellum) (Table 2). Even though only a small number of patients had undergone

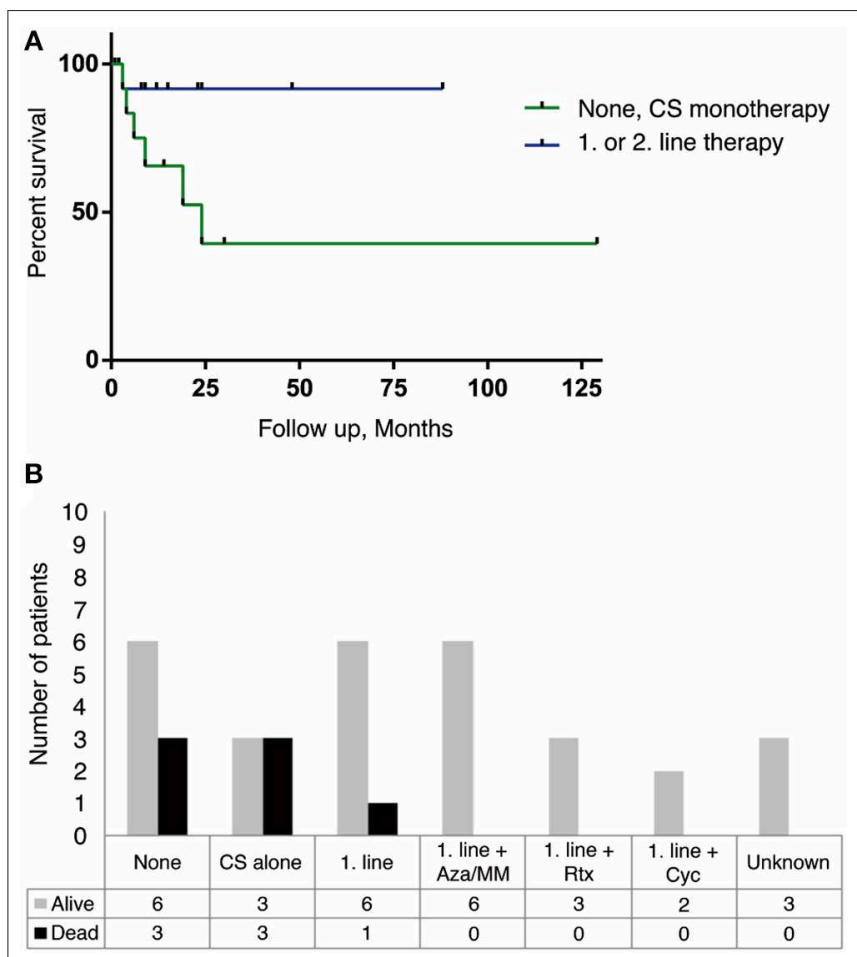

FIGURE 2 | Treatment strategies and survival outcome in anti-IgLON5 disease. Patients receiving no therapy or CS monotherapy had a higher mortality than patients treated with a combination of 1. line therapy (CS+IVIg or TPE or IVIg/TPE alone) and a steroid sparing agent. Similarly, addition of 2. line therapy with Rituximab or cyclophosphamide improved survival. (A) Kaplan-Meier survival curve showing the difference in survival between no therapy and/or CS alone (green line) and 1. or 2. line therapy (blue line). It should be noted that follow-up time differed from case to case, resulting in a high number of censored data (black dot) within an already small population ( $n$ $=27, p=0.064)$. (B) Outcome between different treatment strategies $n=36$. CS, corticosteroids; IVIg, intravenous immunoglobulin; TPE, therapeutic plasma exchange; Aza, Azathioprine; MM, Mycophenolate Mofetil; Rtx, Rituximab; Cyc, Cyclophosphamide. 
brain FDG PET CT, abnormal findings were present in 50\%, confirming the sensitivity of this modality in antibody mediated disease (34). All abnormal findings were hypermetabolism in regions that correlated with clinical symptoms and regions assumed affected by the tauopathy (basal ganglia, brainstem, cerebellum) (Table 2). Leptomeningeal microglial activation was visualized using translocater protein $18 \mathrm{kDa}$ binding (TSPO) PET in one patient with a history of progressive tremor, sleep disturbances and gait impairment, and a tau-PET demonstrated cerebellar tau deposits in the same patient (10).

When performed, PSG was found abnormal in $95 \%$ of cases and symptoms included insomnia, low sleep efficiency, non-REM, and REM parasomnia with finalistic movements and sleep disordered breathing (stridor, respiratory failure, and OSA). Obstructive sleep apnea was present in $64 \%$ of cases (Supplementary Table 1).

$$
\text { Additional ancillary testing including }
$$
electroencephalography, ENG, EMG, DAT SPECT, and whole body FDG-PET CT were mostly unremarkable or nonspecific.

\section{TREATMENT AND OUTCOME}

Overall, majority of patients diagnosed with anti-IgLON5 disease were treated with immunosuppressants $(80 \%)$, but various combinations have been used. The most frequent used were cycles of IV corticosteroids (58\%) in combination with IV immunoglobulins (IVIg-36\%) and/or TPE (27\%). The most commonly used second line treatments were Rituximab (22\%) and Cyclophosphamide (12\%), but other strategies such as Azathioprine and Mycophenolat Mofetil have also been used (5, 7, 10, 17) (Supplementary Table 1). Most of the cases reported have been treated with a combination of at least two different therapy strategies.

The 20 patients reported by Gaig et al., who received therapy, had a median disease duration of 34,5 months and only two of the 20 patients treated with immunotherapy had partial/transient effect of treatment. In the remaining 36 of reported cases, median disease duration was 12 months, and 24 cases received immunotherapy (in three cases, therapy was unknown). Seven of the 36 patients died.

In cases where information about treatment was available, we found no clear correlation between disease duration and disability or death. However, patients who received no treatment or treatment with corticosteroid alone appear to have a higher mortality compared to patients receiving more potent immunotherapy. Six of the seven patients that died, received either IV steroids as monotherapy or no therapy (Figure 2). There was no clear correlation found between therapeutic strategy and degree of remaining disability in patients (reported and estimated mRS scores).

Overall, 20 out of 58 patients with definite anti-IgLON5 disease have been reported dead (34\% mortality). The most common cause of death was sudden death (56\%) followed by aspiration (44\%). Death showed no clear correlation to treatment response, as even cases with partial response died suddenly $(9,14$, 18) (Supplementary Table 1).

Symptomatic treatment with CPAP in patients with OSA improves respiratory symptoms, but has no convincing effect on parasomnias (20). In some patients with movement disorders (myoclonus, parkinsonism, and dystonia) antiepileptic, dopaminergic, and anti-hyperkinetic drugs were administered, but only with sparse effect on symptoms $(7,18,19,33)$.

\section{CONCLUSION}

Anti-IgLON5 disease should be suspected in patients displaying sleep disorder characterized by insomnia, non-REM parasomnia, finalistic movements, and sleep disordered breathing in combination with bulbar symptoms, gait instability, involuntary movements, ocular abnormalities, neuropsychiatric symptoms, dysautonomia, and peripheral nervous system involvement. Antibodies against IgLON5 are crucial for diagnosis, and are present in serum and in almost all cases in CSF. HLADRB1*10:01 and HLA-DQB1*05:01 is strongly associated to presence of anti-IgLON5 antibodies. Brain FDG-PET CT is abnormal in $50 \%$ of cases, and could be more sensitive than MRI. Tau level in CSF, tau-PET or brain biopsy might support the diagnosis, but still needs further exploration. Aggressive immunotherapy seems to be crucial for outcome, as untreated patients or patients treated with steroid monotherapy appear to have a higher mortality. Further studies in larger cohorts with long-term follow up are needed.

\section{DATA AVAILABILITY STATEMENT}

All datasets generated for this study are included in the manuscript/Supplementary Files.

\section{ETHICS STATEMENT}

Ethical review and approval was not required for the study on human participants in accordance with the local legislation and institutional requirements. The patients/participants provided their written informed consent to participate in this study. Written informed consent was obtained from the individual(s) for the publication of any potentially identifiable images or data included in this article.

\section{AUTHOR CONTRIBUTIONS}

MN and MB: design and draft of the manuscript, acquisition and interpretation of data, revised manuscript for intellectual content.

\section{SUPPLEMENTARY MATERIAL}

The Supplementary Material for this article can be found online at: https://www.frontiersin.org/articles/10.3389/fneur. 2019.01056/full\#supplementary-material 


\section{REFERENCES}

1. Sabater L, Gaig C, Gelpi E, Bataller L, Lewerenz J, Torres-Vega E, et al. A novel non-rapid-eye movement and rapid-eye-movement parasomnia with sleep breathing disorder associated with antibodies to IgLON5: a case series, characterisation of the antigen, and post-mortem study. Lancet Neurol. (2014) 13:575-86. doi: 10.1016/S1474-4422(14)70051-1

2. Sabater L, Planaguma J, Dalmau J, Graus F. Cellular investigations with human antibodies associated with the anti-IgLON5 syndrome. J Neuroinflammation. (2016) 13:226. doi: 10.1186/s12974-016-0689-1

3. Gelpi E, Hoftberger R, Graus F, Ling H, Holton JL, Dawson T, et al. Neuropathological criteria of anti-IgLON5-related tauopathy. Acta Neuropathol. (2016) 132:531-43. doi: 10.1007/s00401-016-1591-8

4. Gaig C, Graus F, Compta Y, Hogl B, Bataller L, Bruggemann N, et al. Clinical manifestations of the anti-IgLON5 disease. Neurology. (2017) 88:1736-43. doi: 10.1212/WNL.0000000000003887

5. Honorat JA, Komorowski L, Josephs KA, Fechner K, St. Louis EK, Hinson SR, et al. IgLON5 antibody: neurological accompaniments and outcomes in 20 patients. Neurol Neuroimmunol Neuroinflamm. (2017) 4:e385. doi: 10.1212/NXI.0000000000000385

6. Zhang W, Niu N, Cui R. Serial 18F-FDG PET/CT findings in a patient with IgLON5 encephalopathy. Clin Nucl Med. (2016) 41:787-8. doi: 10.1097/RLU.0000000000001339

7. Haitao R, Yingmai $Y$, Yan $H$, Fei $H$, Xia L, Honglin $H$, et al. Chorea and parkinsonism associated with autoantibodies to IgLON5 and responsive to immunotherapy. J Neuroimmunol. (2016) 300:9-10. doi: 10.1016/j.jneuroim.2016.09.012

8. Haitao R, Huiqin L, Tao Q, Xunzhe Y, Xiaoqiu S, Wei L, et al. Autoimmune encephalitis associated with vitiligo? J Neuroimmunol. (2017) 310:14-6. doi: 10.1016/j.jneuroim.2017.05.019

9. Schroder JB, Melzer N, Ruck T, Heidbreder A, Kleffner I, Dittrich $\mathrm{R}$, et al. Isolated dysphagia as initial sign of anti-IgLON5 syndrome. Neurol Neuroimmunol Neuroinflamm. (2017) 4:e302. doi: 10.1212/NXI.0000000000000302

10. Schoberl F, Levin J, Remi J, Goldschagg N, Eren O, Okamura N, et al. IgLON5: a case with predominant cerebellar tau deposits and leptomeningeal inflammation. Neurology. (2018) 91:180-2. doi: 10.1212/WNL.0000000000005859

11. Bonello M, Jacob A, Ellul MA, Barker E, Parker R, Jefferson S, et al. IgLON5 disease responsive to immunotherapy. Neurol Neuroimmunol Neuroinflamm. (2017) 4:e383. doi: 10.1212/NXI.0000000000000383

12. Morales-Briceno $\mathrm{H}$, Cruse $\mathrm{B}$, Fois AF, Lin MW, Jiang J, Banerjee $\mathrm{D}$, et al. IgLON5-mediated neurodegeneration is a differential diagnosis of CNS Whipple disease. Neurology. (2018) 90:1113-5. doi: 10.1212/WNL.0000000000005679

13. Vetter E, Olmes DG, Linker R, Seifert F. Teaching video neuroimages: facial myokymia and myorhythmia in anti-IgLON5 disease: the bitten lip. Neurology. (2018) 91:e1659. doi: 10.1212/WNL.0000000000006388

14. Wenninger S. Expanding the clinical spectrum of IgLON5-syndrome. $J$ Neuromuscul Dis. (2017) 4:337-9. doi: 10.3233/JND-170259

15. Ramanan VK, Crum BA, McKeon A. Subacute encephalitis with recovery in IgLON5 autoimmunity. Neurol Neuroimmunol Neuroinflamm. (2018) 5:e485. doi: 10.1212/NXI.0000000000000485

16. Tao Q-Q, Wei Q, Song S-J, Yin X-Z. Motor neuron disease-like phenotype associated with anti-IgLON5 disease. CNS Neurosci Ther. (2018) 24:1305-8. doi: $10.1111 /$ cns. 13038

17. Montagna M, Amir R, De Volder I, Lammens M, Huyskens J, Willekens B. IgLON5-associated encephalitis with atypical brain magnetic resonance imaging and cerebrospinal fluid changes. Front Neurol. (2018) 9:329. doi: 10.3389/fneur.2018.00329

18. Bahtz R, Teegen B, Borowski K, Probst C, Blöcker I-M, Fechner K, et al. Autoantibodies against IgLON5: two new cases. J Neuroimmunol. (2014) 275:8. doi: 10.1016/j.jneuroim.2014.08.027
19. Logmin K, Moldovan AS, Elben S, Schnitzler A, Groiss SJ. Intravenous immunoglobulins as first-line therapy for IgLON5 encephalopathy. J Neurol. (2019) 266:1031-3. doi: 10.1007/s00415-019-09221-3

20. Gaig C, Iranzo A, Santamaria J, Graus F. The sleep disorder in anti-lgLON5 disease. Curr Neurol Neurosci Rep. (2018) 18:41. doi: 10.1007/s11910-018-0848-0

21. Hasselbacher K, Steffen A, Wandinger KP, Bruggemann N. IgLON5 antibodies are infrequent in patients with isolated sleep apnea. Eur J Neurol. (2018) 25:e46-7. doi: 10.1111/ene. 13566

22. Mangesius S, Sprenger F, Hoftberger R, Seppi K, Reindl M, Poewe W. IgLON5 autoimmunity tested negative in patients with progressive supranuclear palsy and corticobasal syndrome. Parkinsonism Relat Disord. (2017) 38:102-3. doi: 10.1016/j.parkreldis.2017.03.002

23. Ranaivoson FM, Turk LS, Ozgul S, Kakehi S, von Daake S, Lopez N, et al. A proteomic screen of neuronal cell-surface molecules reveals IgLONs as structurally conserved interaction modules at the synapse. Structure. (2019) 27:893-906.e9. doi: 10.1016/j.str.2019.03.004

24. Karagogeos D. Neural GPI-anchored cell adhesion molecules. Front Biosci. (2003) 8:s1304-20. doi: 10.2741/1214

25. Pimenta AF, Zhukareva V, Barbe MF, Reinoso BS, Grimley C, Henzel W, et al. The limbic system-associated membrane protein is an Ig superfamily member that mediates selective neuronal growth and axon targeting. Neuron. (1995) 15:287-97. doi: 10.1016/0896-6273(95)90034-9

26. Hashimoto T, Maekawa S, Miyata S. IgLON cell adhesion molecules regulate synaptogenesis in hippocampal neurons. Cell Biochem Funct. (2009) 27:496-8. doi: $10.1002 / \mathrm{cbf} .1600$

27. Kubick N, Brosamle D, Mickael ME. Molecular evolution and functional divergence of the IgLON family. Evol Bioinformatics Online. (2018) 14:1176934318775081. doi: 10.1177/1176934318775081

28. Liu F, Arias-Vasquez A, Sleegers K, Aulchenko YS, Kayser M, SanchezJuan P, et al. A genomewide screen for late-onset Alzheimer disease in a genetically isolated Dutch population. Am J Hum Genet. (2007) 81:17-31. doi: $10.1086 / 518720$

29. Karis K, Eskla KL, Kaare M, Taht K, Tuusov J, Visnapuu T, et al. Altered expression profile of IgLON family of neural cell adhesion molecules in the dorsolateral prefrontal cortex of schizophrenic patients. Front Mol Neurosci. (2018) 11:8. doi: 10.3389/fnmol.2018.00008

30. Koneczny I. A new classification system for IgG4 autoantibodies. Front Immunol. (2018) 9:97. doi: 10.3389/fimmu.2018.00097

31. Cagnin A, Mariotto S, Fiorini M, Gaule M, Bonetto N, Tagliapietra M, et al. Microglial and neuronal TDP-43 pathology in anti-IgLON5-related tauopathy. J Alzheimers Dis. (2017) 59:13-20. doi: 10.3233/JAD-170189

32. Balint B, Bhatia KP. Friend or foe? IgLON5 antibodies in a novel tauopathy with prominent sleep movement disorder, ataxia, and chorea. Mov Disord. (2014) 29:989. doi: 10.1002/mds.25926

33. Bruggemann N, Wandinger KP, Gaig C, Sprenger A, Junghanns K, Helmchen $\mathrm{C}$, et al. Dystonia, lower limb stiffness, and upward gaze palsy in a patient with IgLON5 antibodies. Mov Disord. (2016) 31:762-4. doi: 10.1002/mds.26608

34. Solnes LB, Jones KM, Rowe SP, Pattanayak P, Nalluri A, Venkatesan A, et al. Diagnostic value of $18 \mathrm{~F}-\mathrm{FDG}$ PET/CT versus MRI in the setting of antibody specific autoimmune encephalitis. J Nucl Med. (2017) 58:1307-13. doi: $10.2967 /$ jnumed.116.184333

Conflict of Interest: The authors declare that the research was conducted in the absence of any commercial or financial relationships that could be construed as a potential conflict of interest.

Copyright $\odot 2019$ Nissen and Blaabjerg. This is an open-access article distributed under the terms of the Creative Commons Attribution License (CC BY). The use, distribution or reproduction in other forums is permitted, provided the original author(s) and the copyright owner(s) are credited and that the original publication in this journal is cited, in accordance with accepted academic practice. No use, distribution or reproduction is permitted which does not comply with these terms. 